\title{
Traffic speed deflectometer measurements at Copenhagen airport
}

\author{
C.P. Nielsen \& K. Jensen \\ Greenwood Engineering A/S, Copenhagen, Denmark
}

\begin{abstract}
The results from a Traffic Speed Deflectometer (TSD) measurement campaign in Copenhagen airport are presented. The structural condition was measured with high spatial resolution on one runway with flexible pavement and one runway with concrete slabs overlaid with asphalt. The runway with flexible pavement had a high stiffness, and comparisons with HWD measurements showed a good agreement between TSD measurements and HWD measurements. On the runway with asphalt overlaid concrete slabs, the joint movement was quantified based on the TSD measurements. This revealed areas with comparatively high joint movement, which could be susceptible to reflective cracking of the asphalt layer. A total of $189 \mathrm{~km}$ runway was measured in four hours.
\end{abstract}

Keywords: Traffic Speed Deflectometer, TSD, Structural condition, Airport pavement, Runway pavement

\section{INTRODUCTION}

The structural condition of an airport's runways is of critical importance to the operation of the airport. Unexpected runway breakdowns can lead to substantial economic losses for the airport, and can affect the wider community by disrupting mobility. For these reasons, many airports perform routine Heavy Weight Deflectometer (HWD) surveys of runway structural condition (AC 2016; Pigozzi et al. 2015). The slow measuring speed of the HWD does, however, pose a challenge for its use on airport runways. The runway needs to be closed for an extended period of time while the measurements are done, and time constraints make it impractical to measure along the runway in more than a few lines.

In the road sector, similar challenges have led to the adoption of the Traffic Speed Deflectometer (TSD) for surveying structural condition. The TSD uses an innovative principle based on Doppler lasers to measure the response of the pavement to a 10 tonne axle, while moving at ordinary traffic speed. While the early TSDs were mainly used as screening devices and a supplement to FWD measurements, the newest TSD generations deliver data of a very high quality which can be used for work at the project level. Due to these improvements in measurement accuracy, it is now feasible to perform TSD measurements under the comparatively challenging conditions posed by an airport runway.

In this paper we present results from a TSD measurement campaign in Copenhagen Airport. Two different runways were measured during the campaign; one runway with flexible pavement and one runway with asphalt overlaid concrete slabs. The structural condition of one runway was measured with a high spatial resolution in both the longitudinal and the transverse direction. The other runway was measured with high spatial resolution in the longitudinal direction. 


\section{THE TRAFFIC SPEED DEFLECTOMETER}

The Traffic Speed Deflectometer is a nondestructive pavement evaluation device, which uses laser Doppler vibrometers to measure the pavement response to a moving 10 tonne axle. The Doppler lasers measure the deflection velocity of the pavement surface at several points on the line passing between the center of the truck's twin tires. Combining this with a measurement of the driving speed, the slope of the deflection basin is recovered. The slope of the deflection basin can be used directly as a pavement condition indicator, or a backcalculation algorithm can be applied to find the elastic moduli of the pavement layers. The TSD used in this measurement was instrumented with 11 Doppler lasers; 8 in front of the load and 3 behind the load. See Refs. (Krarup et al. 2006; Nielsen 2019) for more information.

The TSD measurements took place on the $7^{\text {th }}$ of May 2020 at Copenhagen Airport. Over the course of the measurements the air temperature varied between $11.2^{\circ} \mathrm{C}$ and $16.0{ }^{\circ} \mathrm{C}$, and the runway surface temperature varied between $14.0^{\circ} \mathrm{C}$ and $22.0^{\circ} \mathrm{C}$. The measurements were made with a driving speed close to $80 \mathrm{~km} / \mathrm{h}$.

\section{MEASUREMENTS ON RUNWAY 04R-22L}

Runway $04 \mathrm{R}-22 \mathrm{~L}$ is one of the two main runways of Copenhagen airport. The $04 \mathrm{R}$ direction is mainly used for aircraft take off, and is responsible for $32 \%$ of the take offs in Copenhagen airport. The $22 \mathrm{~L}$ direction is mainly used for landings, and is responsible for $60 \%$ of the landings. The runway has a length of $3.3 \mathrm{~km}$ and is paved with $350 \mathrm{~mm}$ asphalt on top of a $300 \mathrm{~mm}$ unbound base layer. The runway was measured from edge to edge (including shoulders) with 48 passes of the TSD.

The performance of the top part of the pavement can be quantified using the surface curvature index $\mathrm{SCI}_{300}$, which is defined as the difference in deflection between the load position and a position $300 \mathrm{~mm}$ from the load (Pigozzi et al. 2015). The curvature of the deflection basin is related to the horizontal strain in the bottom of the asphalt layer, which is an indicator of the pavement's susceptibility to fatigue damage. In Figure 1 the $\mathrm{SCI}_{300}$ values measured with the TSD are plotted on top of a satellite image of runway 04R-22L. The reported $\mathrm{SCI}_{300}$ values are averaged in sections of one meter. The scale of the colorbar is chosen so that it spans the range of most of the values encountered on the runway proper. The $\mathrm{SCI}_{300}$ values encountered on the shoulders are significantly higher than the chosen color scale, and the shoulders are therefore shown as yellow in most places. The notable exceptions are the places where taxiways lead into the runway: in these places the $\mathrm{SCI}_{300}$ values on the shoulder become comparable to the values on the runway. The $\mathrm{SCI}_{300}$ values found on the runway are all very low, in the range $5 \mu \mathrm{m}$ to $25 \mu \mathrm{m}$, showing that it is a very stiff pavement. Nevertheless, some structure in the reported values is apparent. Around $x=-800 \mathrm{~m}$ a large area of comparatively high $\mathrm{SCI}_{300}$ is visible on both sides of the runway. These areas were initially thought to coincide with the touch down zone for aircraft landing in the 04R direction. However, only $2 \%$ of the airplanes land in the $04 \mathrm{R}$ direction and the designated touch down zone marked on the runway is located near to $x=-1500 \mathrm{~m}$. The reason for these large areas with higher $\mathrm{SCI}_{300}$ is therefore not readily apparent. It is probably a combination of usage patterns, small irregularities in the original construction and the maintenance history of the runway which has led to the observed behavior. The same is the case for the other regions of high or low $\mathrm{SCI}_{300}$, which can be observed in Figure 1. It should, however, be stressed that the yellow areas are only weak in comparison. In absolute terms the entire runway is very stiff. 


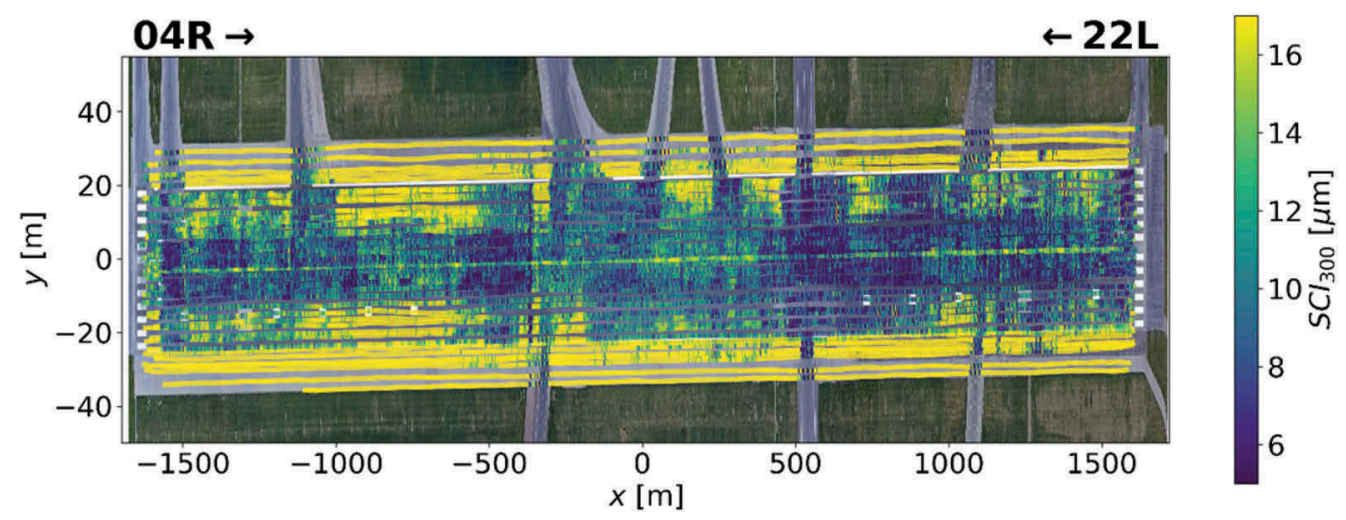

Figure 1. $S C I_{300}$ values measured with the TSD on runway 04R-22L reported in $1 \mathrm{~m}$ sections.

\subsection{Back-calculation on runway 04R-22L}

The $\mathrm{SCI}_{300}$ index is convenient for getting a quick overview of the pavement performance. To quantify the pavement performance in more detail, the measurements were processed using a viscoelastic back-calculation algorithm developed specifically for the TSD, see Ref. (Nielsen 2019). The outputs of the back-calculation are the elastic moduli of the pavement layers and the pavement strains evaluated at points of interest in the pavement structure. In the backcalculation a layer thickness of $350 \mathrm{~mm}$ was used for the asphalt layer and $300 \mathrm{~mm}$ was used for the unbound base layer. The subgrade was assumed to be an infinite half-space. Before applying the back-calculation algorithm, the measurements were averaged in $10 \mathrm{~m}$ sections. In Figure 2 the back-calculated modulus of the asphalt layer on runway 04R-22L is plotted. In general, the asphalt is seen to be quite stiff, having elastic moduli in the range from $5000 \mathrm{MPa}$ to $25000 \mathrm{MPa}$. It is, however, also seen that the behavior is not entirely uniform; within the yellow areas of high modulus there are small blue sections of lower modulus. These blue spots could indicate actual areas of reduced modulus, but on closer inspection it becomes apparent that they are actually areas where the back-calculation fits the data poorly. The reason for these poor fits is that the layer thicknesses used in the back-calculation do not accurately describe the pavement. Most likely, the $350 \mathrm{~mm}$ asphalt layer is composed of many different asphalt layers applied over the course of the runway's lifetime. Over time, the layers in the bottom have lost much of their stiffness, whereas the layers in the top still retain a high elastic modulus. This assumption was in fact confirmed by asphalt cores, which had previously been taken by airport maintenance personnel. The cores showed a reduced asphalt quality in the lower parts of the $350 \mathrm{~mm}$ asphalt layer. In Figure 3 some examples of TSD measurements and model fits are shown with an assumed top layer thickness of $350 \mathrm{~mm}$ (blue) and $150 \mathrm{~mm}$ (orange). It is seen that the model with $150 \mathrm{~mm}$ captures the measured behavior much better than the model with $350 \mathrm{~mm}$. Nevertheless, to be consistent with the existing HWD backcalculations on the runway, the model with $350 \mathrm{~mm}$ asphalt is used in the following.

One of the main motivations for making a back-calculation is that it allows for an evaluation of the critical strains in the pavement structure. In Figure 4 the back-calculated horizontal strain $\epsilon_{x x}$ in the bottom of the asphalt layer is plotted. This strain is an important indicator, since it is related to the development of fatigue cracking. It is seen that the backcalculated horizontal strain follows the same pattern as the surface curvature index in Figure 1. It is important to note, that although the issues with the asphalt layer thickness discussed above will have an adverse effect on the quality of the modulus estimates, the strain estimates are still expected to be reasonably good. The reason for this is, that knowledge of the surface deflections puts some bounds on the possible values of the strains. Thus, even if there are some errors in the asphalt modulus, the estimated strain cannot deviate much from the true strain. This point is treated in more detail in (Nielsen 2020), where it is also shown 


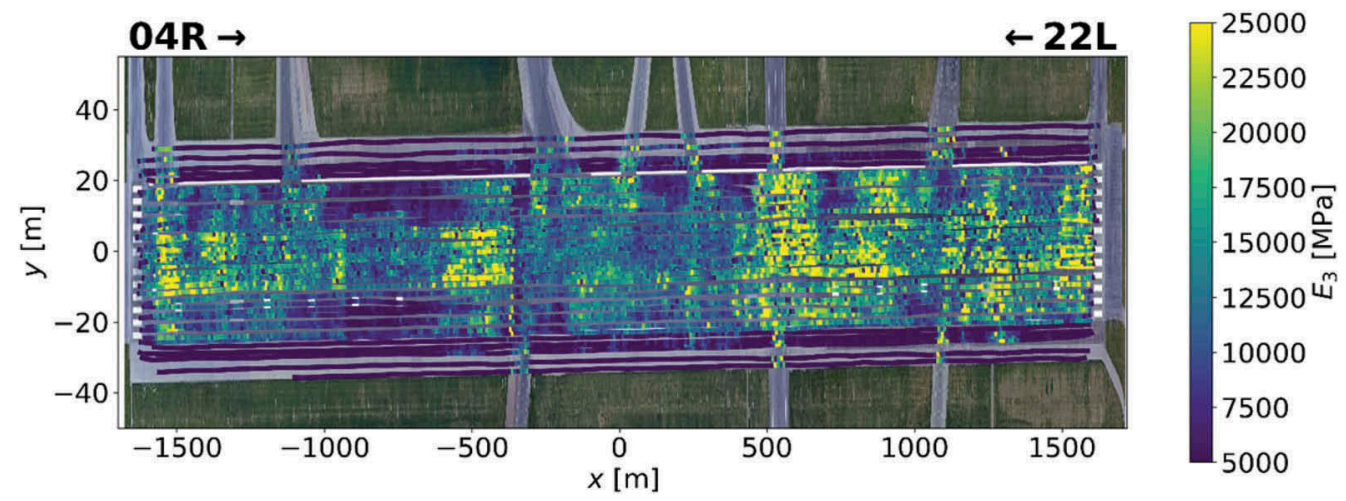

Figure 2. Back-calculated elastic moduli of the asphalt layer on runway 04R-22L.
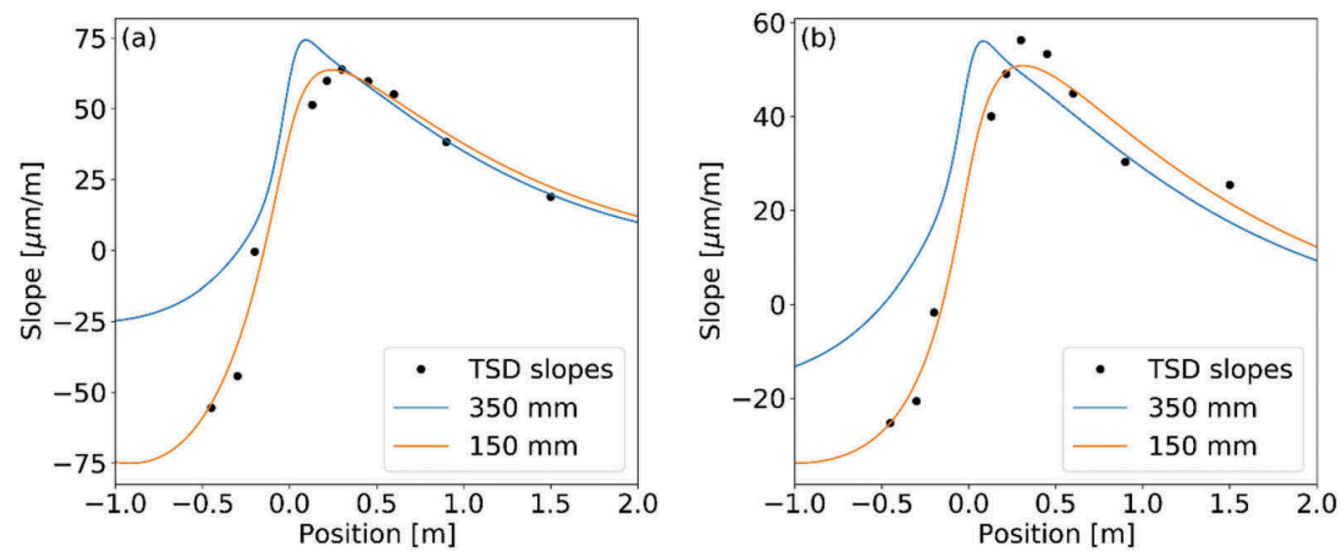

Figure 3. Two examples of measured and fitted slopes. The blue line assumes a top layer thickness of $350 \mathrm{~mm}$ and the orange line assumes a top layer thickness of $150 \mathrm{~mm}$.

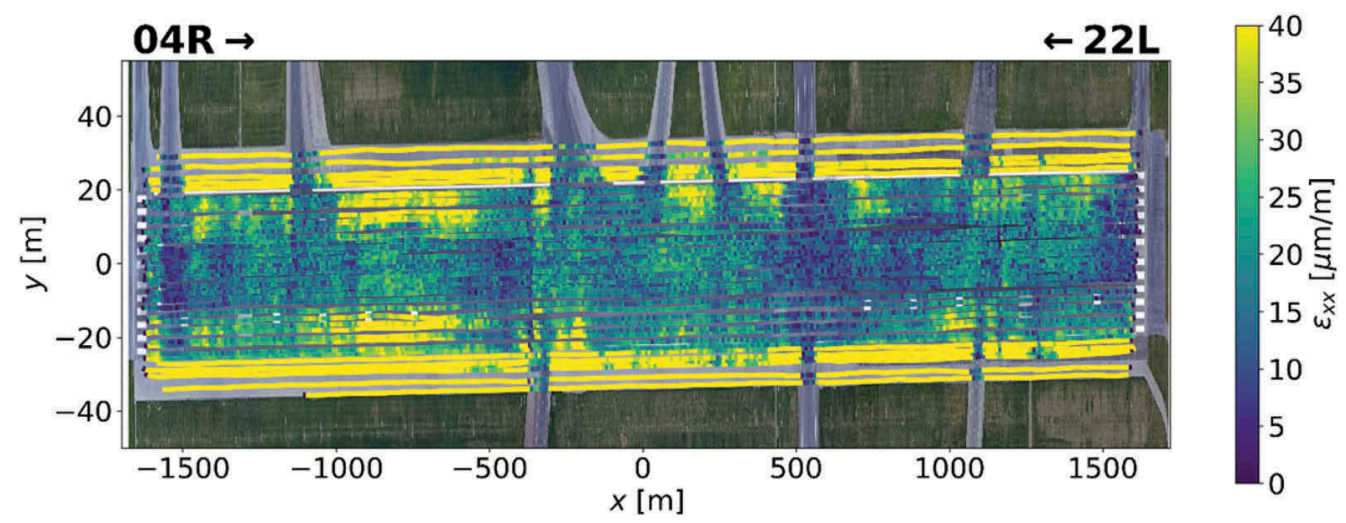

Figure 4. Back-calculated horizontal strain $\epsilon_{x x}$ in the bottom of the asphalt layer on runway 04R-22L. 
that good estimates for the pavement strains can often be obtained without any knowledge of the elastic moduli.

\subsection{Comparison with HWD measurements}

The structural condition of runway 04R-22L had previously been measured with an HWD on October $5^{\text {th }}$ 2017. The measurements were made with a spacing of $60 \mathrm{~m}$ and at each measurement position three different load levels $(100 \mathrm{kN}, 150 \mathrm{kN}, 220 \mathrm{kN})$ were used. To avoid disrupting the runway operation, the measurements were made in the night with a runway surface temperature around $8{ }^{\circ} \mathrm{C}$. Comparing the deflections obtained with each load level revealed that the deflections had a close to linear dependence on the load. It should therefore be possible to compare the HWD measurements with the TSD measurements by scaling the measurements with the used load. This also agrees with the findings from sensitivity studies at the National Airport Pavement Test Facility (AC 2016). In Figure 5 the $\mathrm{SCI}_{300}$ index measured by the TSD is plotted together with the $\mathrm{SCI}_{300}$ index measured by the HWD. The HWD values have been scaled with the ratio of the loads used by the two devices. The $\mathrm{SCI}_{300}$ values from the two devices are seen to be comparable, although the patterns do not match completely. In Figure 6 (a) the $\mathrm{SCI}_{300}$ values are plotted for the topmost HWD line together with the TSD values for the two lines which fall closest to the HWD line. Again, the HWD values are scaled with the relative loads. The TSD measurements were made close to each other, but they are not coinciding exactly. Therefore, the two TSD measurements deviate a little from each other. The scaled HWD measurements are seen to follow the same pattern as the TSD measurements, and the magnitude of the values have a good agreement with those obtained from the TSD. There are multiple possible explanations for the small discrepancies which are observed between the TSD and HWD results. 1: The HWD and TSD measurements were not made in exactly the same spots, and we should expect some deviation on that account. 2: The HWD measurements were made three years before the TSD measurements, and at a different pavement temperature. 3: The loading levels for the two devices were different, and if the subgrade behaves nonlinearly this could lead to a difference in the responses. This is, however, believed to be a small factor for the pavement at hand, since the HWD measurements revealed a close to linear dependence on the load. 4: The HWD and TSD have different loading mechanisms. The HWD simulates a moving load, by applying an impulsive load of a certain duration. The TSD, on the other hand, measures the pavement response to an actual moving load. Using an impulsive load to simulate a moving load will lead to an error if the pavement behaves viscoelastically. Since the runway pavement has a relatively thick asphalt layer, we would expect viscoelasticity to have a quite significant effect on the pavement behavior. The HWD measurements could therefore be significantly skewed by the used loading mechanism. Overall, however, the measurements from the two devices are seen to match quite well.

In Figure 6 (b) the back-calculated elastic moduli of the asphalt layer based on both devices are plotted. There is seen to be an appreciable difference between the values obtained on the two TSD lines. This is partly due to small differences in driving path, but also, and more significantly, due to the layer thickness assumed in the back-calculation. Nevertheless, the TSD and HWD based moduli are seen to be of similar magnitude and exhibit some of the same patterns.

It is seen that the HWD moduli in Figure 6 (b) contain one point with a very low modulus. The corresponding $\mathrm{SCI}_{300}$ point has a value near $100 \mu \mathrm{m}$ and is therefore not visible in Figure 6 (a). The obvious interpretation is that this point is an outlier caused by a measurement error, and that it should be disregarded from the evaluation of the runway. This is, however, only an assumption. Conceivably, there could be a small region at this location, with a much-reduced pavement stiffness. Based only on the sparsely sampled HWD measurements, it is not possible to tell which of these possibilities is true. In contrast, the high spatial resolution of the TSD measurements allows all the stiffness variations to be visualized, and makes it easy to spot whether an outlier has occurred. 


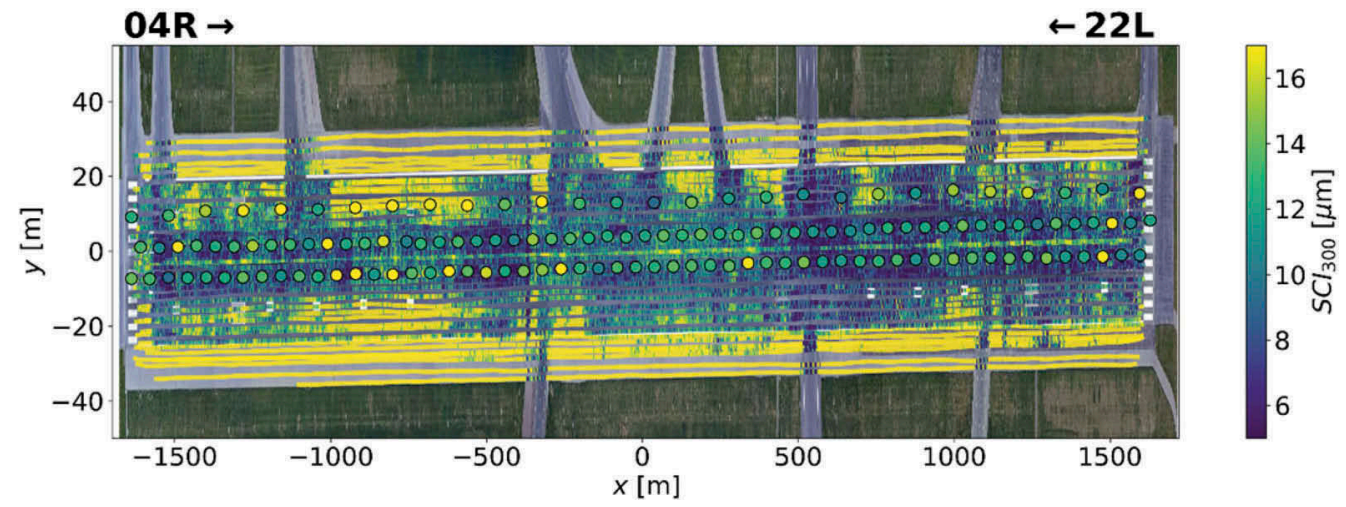

Figure 5. $S C_{300}$ measured with the TSD (lines) and measured with the HWD (dots). The HWD measurements have been scaled by the relative loads to allow for a direct comparison.
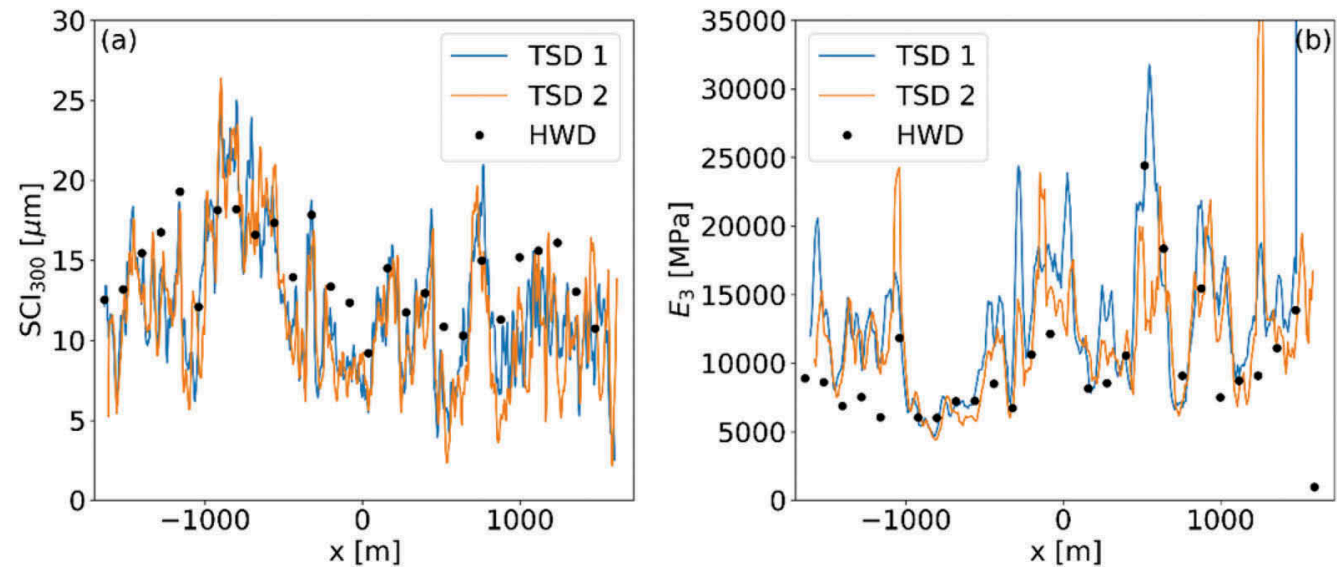

Figure 6. 1D comparison between TSD and HWD measurements. (a): $S C I_{300}$ measured with the TSD (lines, $10 \mathrm{~m}$ moving average) and measured with the HWD (dots). The HWD measurements have been scaled by the relative loads to allow for a direct comparison. (b): Back-calculated elastic moduli of the asphalt layer based on the TSD measurements (lines, $40 \mathrm{~m}$ moving average) and the HWD measurements (dots).

\section{MEASUREMENTS ON RUNWAY 12-30}

Runway 12-30 runs orthogonally to the two main runways and is used much less frequently. The 12 direction accounts for $0.9 \%$ of all landings and take offs, and the 30 direction accounts for $4.7 \%$ of the landings. Originally, the runway was composed of concrete slabs, which are still visible on the shoulders and in the 30 end of the runway. On the remaining part of the runway, the concrete slabs have been overlaid with asphalt.

Measuring on a concrete pavement is different from measuring on an ordinary flexible pavement. The concrete slabs are almost always very stiff, and it is therefore less relevant to measure exactly how stiff they are. The pavement structure is, however, weak at the joints of the concrete slabs. This allows for motion of the slabs, and if this motion is too large it may result in erosion of the base layer or reflective cracking of the asphalt 
overlay. With the TSD it is possible to measure the motion of the concrete slabs, as the loaded axle moves from one slab to the next. This motion is conveniently quantified via the difference in Doppler velocity in front of the load and behind the load $\Delta v=v_{+} v$. For the TSD used in this measurement the $v_{+}$laser is located $13 \mathrm{~cm}$ in front of the load, and the $v_{\text {laser }}$ is located $20 \mathrm{~cm}$ behind.

The runway was measured with 6 passes of the TSD and the data reported in $5 \mathrm{~cm}$ intervals. In Figure 7 (a) the velocity difference $\Delta v$ is plotted along one of the measurements. In Figure 7 (b) a zoom of the same measurement is shown. Spikes in $\Delta v$ are seen at regular intervals, corresponding to the locations of the slab joints. In most of these places the slabs and joints are covered with asphalt, but the joint movement is still clearly visible through the asphalt. In Figure 8 the joint movement is visualized on the surface of the runway. Here, the locations with joint movement have been identified and shown with a dot, and each dot has been colored based to the magnitude of the $\Delta v$ peak. It is seen that there is only visible joint movement on some parts of the runway, and the magnitude of the joint movement varies significantly from region to region. As an example, the region near $x=300 \mathrm{~m}$ and $y=-10 \mathrm{~m}$ contains numerous joints with $\Delta v$ close to $35 \mathrm{~mm} / \mathrm{s}$. This indicates that the region could be prone to reflective cracking. Inspecting satellite images of the runway, it was found that there are signs of reflective crack repairs in that area (also faintly visible in Figure 8). As another example, we consider the region between $x=700 \mathrm{~m}$ and $x=1200 \mathrm{~m}$. Here, the concrete slabs in the middle of the runway were broken into smaller pieces a few years previously. It is seen, that this had the desired effect; there is no joint movement in the middle of the runway. Only the bottom line around $y=-15 \mathrm{~m}$ exhibits joint movement. Evidently, this line is outside the region that was reconstructed.

To put the TSD measurements on runway 12-30 in perspective, measuring the load transfer of every joint with an FWD would require one measurement for every five meters. At one minute per measurement and a runway length of $2.7 \mathrm{~km}$, it would take 9 hours to measure one lane with an FWD. With the TSD, each lane was measured in around two and a half minutes.

We note that the $\Delta v$ index used to quantify the joint movement in this survey, is different from the conventionally used load transfer efficiency (LTE). The two indices are obviously correlated, and in general places with a high $\Delta v$ will have a low LTE. However, the relation between the two indices is not one-to-one. In an upcoming paper on TSD measurements on concrete pavements, the relation between LTE and TSD based load transfer indices is explored further.
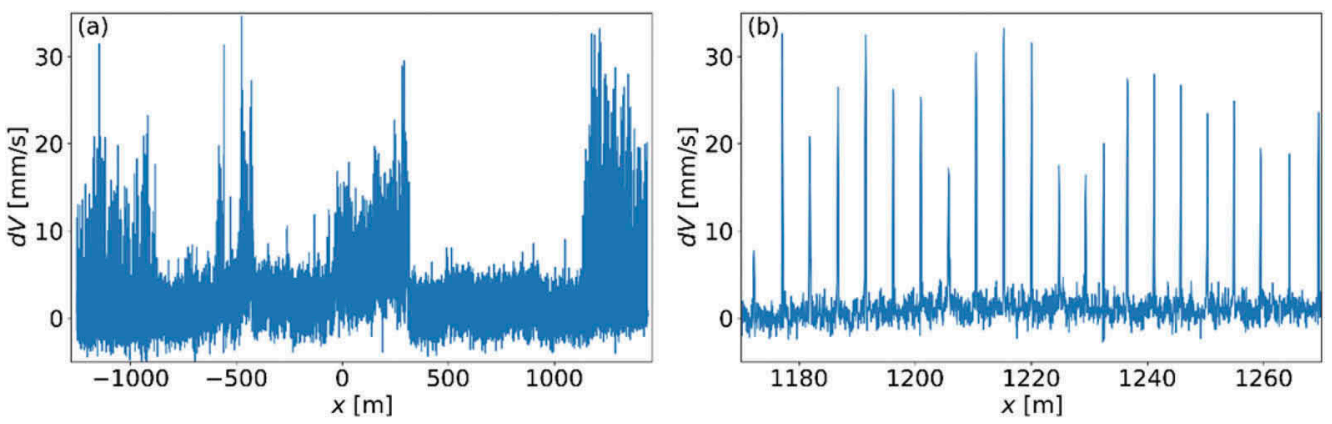

Figure 7. (a): Plot of $\Delta v$ along one measurement on runway 12-30. (b): Zoom of (a). 


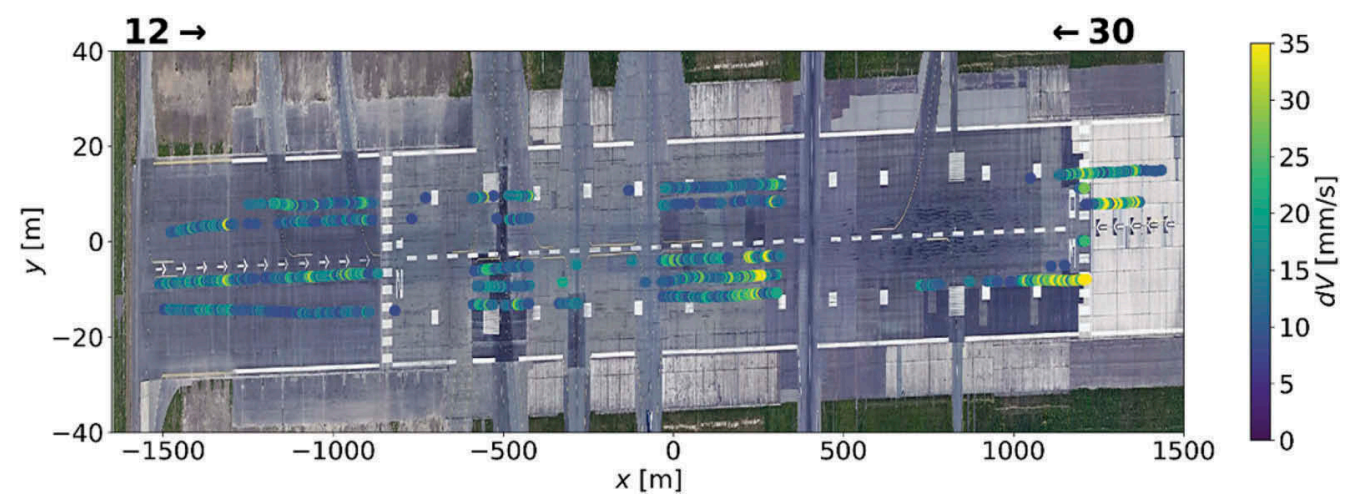

Figure 8. Locations of joint movement on runway 12-30. The color scale shows the magnitude of the joint movement.

\section{SUMMARY AND CONCLUSION}

The ability of the TSD to measure on airport runways was demonstrated on two runways in Copenhagen airport. The measurements on runway 04R-22L proved that the TSD is able to measure on very stiff flexible pavements, and the TSD proved its ability to consistently discriminate between stiff and very stiff areas of the pavement. The $\mathrm{SCI}_{300}$ values measured with the TSD had a good agreement with earlier HWD measurements on the runway. It was, however, found that the layer thicknesses previously used for HWD back-calculation did not represent the pavement structure well. For this reason, the elastic moduli back-calculated based on HWD and TSD did not agree as well as the raw measurements.

The pavement on runway 12-30 was composed of asphalt overlaid concrete slabs, which made it relevant to investigate joint movement rather than pavement stiffness. In large areas of the runway, movement of the slab joints could be detected through the asphalt overlay. Some of these areas could be susceptible to reflective cracks in the asphalt overlay.

In conclusion, the measurements in Copenhagen airport have demonstrated that the TSD is able to measure runway structural condition on two different pavement types, and that it is able to do it rapidly, consistently, and with high spatial resolution.

\section{REFERENCES}

AC. 2016. 150/5320-6F. Airport Pavement Design and Evaluation.

Krarup, J., S. Rasmussen, L. Aagaard, and P. G. Hjorth. 2006. Output from the Greenwood Traffic Speed Deflectometer. In Paper Presented to 22nd ARRB Conference. Canberra, Australia.

Nielsen, C.P. 2019. Visco-Elastic Back-Calculation of Traffic Speed Deflectometer Measurements. Transportation Research Record.

Nielsen, C.P. 2020. Deriving Pavement Deflection Indices from Layered Elastic Theory. Transportation Research Record.

Pigozzi, Franco, Silvia Portas, Francesca Maltinti, and Mauro Coni. 2015. Analysis of Runway Deflectometer Campaign for Implementation on Airport Pavement Management System. International Journal on Pavement Engineering \& Asphalt Technology 15 (2): 11-26. 